\title{
Mathematical modeling of mass transfer in baromembrane processes
}

\section{Oleksandr Obodovich ${ }^{1}$, Oleksandr Ustinov ${ }^{2}$, Volodymyr Zaharov ${ }^{2}$}

\author{
1 - Institute of Engineering Thermophysics of National academy of science of \\ Ukraine, Kyiv, Ukraine \\ 2 - National University of Food Technologies, Kyiv, Ukraine
}

Keywords:

Membrane,

Contamination

Mass transfer

Ultrafiltration,

Modeling

\section{Article history:}

Received 16.04.2018

Received in revised

form 20.06.2018

Accepted 29.06.2018

\section{Corresponding}

author:

Oleksandr Ustinov

E-mail:

hokalextyr@

gmail.com

DOI: $10.24263 / 2304-$

974X-2018-7-2-13

\section{Abstract}

Introduction. To determine the optimal modes of rational exploitation of membranes the phenomena of concentration polarization was studied here.

Materials and methods. Distribution of the concentration of the dissolved substance in solvent by height of the channel of the baromembrane apparatus was obtained by the method of mathematical modeling. Membrane channels were represented as a rectangular grid with a predetermined step. The stability condition of the solutions was verified using the Courant criterion.

Results and discussion. Applying the corresponding boundary conditions characterizing the physical essence of the baromembrane processes, as well as numerical methods for solving differential equations, we obtain a system of algebraic kinetic equations that allow us determine the distribution of concentration of the dissolved substance by the height of the pressure channels, which is practically impossible to do experimentally. To simulate the real processes of separation, we used geometric, physical and mass-exchange characteristics of the real membrane systems. It is established that due to the semi-permeable properties of membranes, the amount of the dissolved substance at its surface over time increases, that is, the phenomenon of concentration polarization appears. Depending on the pressure, membrane characteristics and flow turbulence, the value of the concentration polarization may exceed the value of 10 , which must be taken into account in order to prevent the formation of sediment on the surface of the membrane.

The character of the obtained dependencies is in good agreement with the theoretical foundations of the membrane processes and allows us apply the proposed algorithm for the preliminary analysis of the phenomena occurring in the pressure channels in the division of complex multicomponent liquid systems.

Conclusions. The level of concentration polarization in the membrane channels of barometric apparatuses at separations processes of food industry liquids may exceed 10 and depends upon the parameters of the process. 


\section{Introduction}

The problem of the use of membrane technology lies in the increased level of membrane contamination due to the use of wrong operating modes. As a result, the concentration of solutes at the membrane surface increases significantly (the phenomenon of concentration polarization). At high concentrations, some components may form insoluble compounds, gel-form sediment and other. When the concentration in the layer near surface of membrane becomes higher than the concentration of the feeding solution, then there are diffusion streams directed in the opposite direction relative to the flow of the filtering solution. Such effects significantly reduce the membrane productivity, in the process of separation processes $[1,2]$.

Therefore, it is advisable to investigate the phenomenon of concentration polarization, studying the distribution of concentrations and its change over time, in the channel, to determine the optimal modes of rational operation of membranes. Since experimentally, determining the distribution of concentrations during the filtration process is very difficult, methods of mathematical modeling are used. Unfortunately, there is now no single theory capable of fully describing the processes that take place near the surface of the membrane for the processes of separation (concentration) of multicomponent solutions [3], so this task remains relevant. Among modern works, there are stochastic approaches [4], various semiempirical models $[3,5]$, but in most cases the theoretical study of mass transfer processes is carried out by methods of continuum mechanics using methods of finite-difference approximation $[3,5,6,7]$.

For filtration, mass transfer occurs by two mechanisms: convective transfer and diffusion [8]. Differential equations that can describe the behavior of such systems, in general, have no precise analytical solutions due to their complexity and effect nonlinearity $[3,9,10]$. Therefore, numerical methods such as finite-difference approximation methods are used to obtain approximate solutions $[5,7]$.

This method allows solving the differential equation under given boundary and initial conditions and requires a minimum of information $[5,6]$. Relative simplicity and visibility of the method allows it to be applied very quickly to solve various technological and research problems [3].

The purpose of this work was to determine level of the concentration polarization in the membrane channels of barometric apparatus by methods of mathematical modeling in the separation processes of food industry liquids.

\section{Materials and methods}

The phenomenon of concentration polarization related to mass transfer processes, namely, the distribution of the concentration of $\mathrm{C}(\mathrm{x}, \mathrm{t})$ of the dissolved substance in solvent by height of the channel of baromembrane apparatuses is studied.

Concentration, $\mathrm{kg} / \mathrm{m}^{3}$, is defined as the mass of the target component per unit volume:

$$
C=\frac{d M}{d V}
$$

For one-dimensional models, the concept of linear concentration, $\mathrm{kg} / \mathrm{m}$, is introduced, that is, the mass of the dissolved substance per unit length of the segment of the coordinate $\mathrm{x}$ :

$$
C_{\text {line }}=\frac{d M}{d x}
$$


Then $\mathrm{C}_{\text {line }}=\mathrm{C}_{\text {volume }} \cdot \mathrm{S}$, where $\mathrm{S}$ is the area, $\mathrm{C}_{\text {volume }}$ is the volumetric concentration. The mass flow $\mathrm{q}_{\mathrm{m}}, \mathrm{kg} / \mathrm{s}$, is the mass of the dissolved substance passing through the surface $\mathrm{S}$ per unit time.

The investigated medium is considered in generalized (dimensionless) coordinates (grid nodes are applied) [3,5]. By using the boundary and initial conditions and the first principle of differentiation, the function of concentration can be determined for each point of the space. As a result, the differential equation is approximated by a system of algebraic and at known values of the concentration function at certain points (or at a certain point in time) we can obtain the value of a function at any point of the system under study $[5,6,7]$.

For explicit schemes of finite-difference approximation the stability conditions formulated by the Courant's criterion [6]. The physical essence of this criterion is that the speed of movement along the grid should be less than the transmission rate of perturbations in the system. For this case, the Courant's criterion is determined by the relation:

$$
\Delta t<\frac{\Delta x^{2}}{2 D},
$$

where $\mathrm{D}$ is the diffusion coefficient, $\Delta \mathrm{x}, \Delta \mathrm{t}$ is the spatial and temporal step according to the scheme, respectively.

\section{Results and discussion}

The density of the mass flow $\mathrm{q}_{\mathrm{sm}}, \mathrm{kg} / \mathrm{s} \cdot \mathrm{m}^{2}$, is the mass of the target component passing a unit of the surface over time in a given direction. Although $\mathrm{q}_{\mathrm{Sm}}$ is a vector value, but in one-dimensional models it becomes essentially a scalar: $\mathrm{q}_{\mathrm{m}}=\mathrm{q}_{\mathrm{Sm}} \mathrm{S}$. The main empirical diffusion law is Fica's law:

$$
q_{S m}=-D \frac{d C}{d x},
$$

where $\mathrm{D}$ is the diffusion coefficient, $\mathrm{m}^{2} / \mathrm{s}$. Moreover, he represents the characteristic of the system «dissolved substance - carrier (solvent) ». If the solution moves at a velocity v, then the mass flow (i.e., convective flow) is defined as:

$$
q_{m}^{\text {conv }}=C_{\text {volume }} \cdot S \cdot v=C_{\text {line }} \cdot v=C \cdot v,
$$

And formally coincides with the density of the flow. In the study of one-dimensional models, it should be borne in mind that $\mathrm{C} \cdot \mathrm{v}$ is the flow, not its density. Similarly, for a diffusion stream of mass (at $\mathrm{S}=$ const):

$$
q_{m}^{\text {diff }}=q_{S m}^{\text {diff }} \cdot S=-D \frac{\partial C_{\text {volume }}}{\partial x} \cdot S=-D \frac{\partial C}{\partial x},
$$




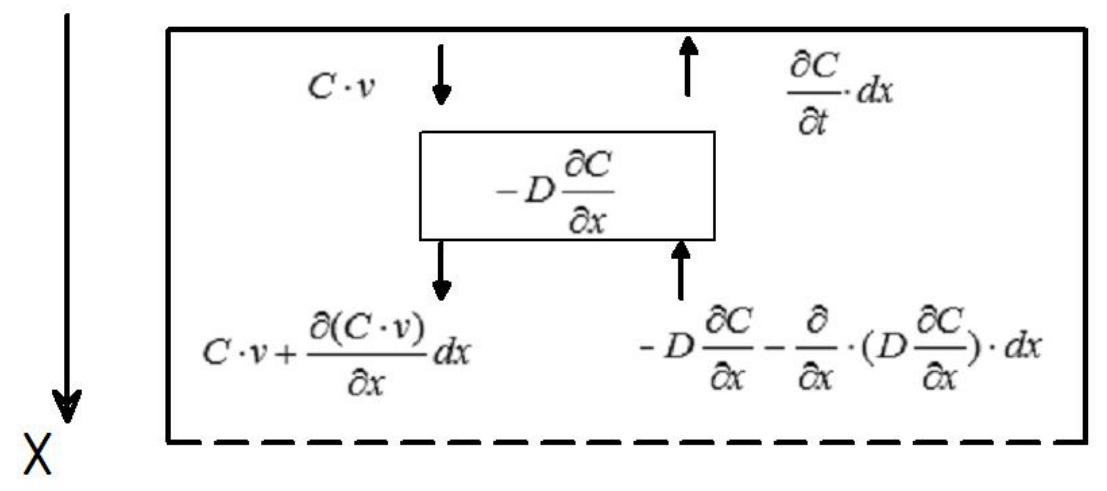

Figure 1. Scheme of flows in the membrane channel: $X$-spatial coordinate.

$\mathrm{C}=\mathrm{C}(\mathrm{x}, \mathrm{t})$ - concentration of the dissolved substance at the point $\mathrm{x}$, at time $\mathrm{t}, \mathrm{kg} / \mathrm{m}$. Change of its mass in volume $\mathrm{S} \cdot \mathrm{dx}$ for time dt equals $\frac{\partial C}{\partial t} \cdot d x$. As a result of the movement of convective and diffusion streams, the concentration of the target component in the considered elemental volume also changes (Figure 1).

Diffusive flows:

$$
\begin{gathered}
q_{1}^{d i f f}=-D \frac{\partial C}{\partial x} \\
q_{2}^{d i f f}=-D \frac{\partial C}{\partial x}-\frac{\partial}{\partial x}\left(D \frac{\partial C}{\partial x}\right) d x,
\end{gathered}
$$

Convective flows:

$$
\begin{gathered}
q_{1}^{\text {conv }}=C \cdot v, \\
q_{2}^{\text {conv }}=C \cdot v+\frac{\partial(C \cdot v)}{\partial x} d x,
\end{gathered}
$$

$\mathrm{q}_{\mathrm{e}} \mathrm{dx}$ - mass flow from external sources, $\mathrm{kg} / \mathrm{s}, \mathrm{q}_{\mathrm{e}}$ - flow density, $\mathrm{kg} / \mathrm{m} \mathrm{s}$.

Taking into account the balance of masses in the allocated volume we obtain the equation of convective diffusion with an external source:

$$
\frac{\partial C}{\partial t}=-\frac{\partial(C \cdot v)}{\partial x}+\frac{\partial}{\partial x}\left(\frac{D \cdot \partial C}{\partial x}\right)+q_{e},
$$

where $\mathrm{v}, \mathrm{D}, \mathrm{q}_{\mathrm{e}}$ are known functions and parameters. In the general case, this is a nonlinear equation in partial derivatives with variable coefficients. But the classical form of the equation has $\mathrm{v}=$ const, $\mathrm{D}=$ const, $\mathrm{q}_{\mathrm{e}}=0$ :

$$
\frac{\partial C}{\partial t}=-V \frac{\partial C}{\partial x}+D \frac{\partial^{2} C}{\partial x^{2}}
$$

The solution $C=C(x, t)$ must satisfy the initial and boundary conditions set at the boundaries of the interval $[0, h]$ at the points $x=0, x=h$ and set the upper boundary of the channel and the surface of the membrane. $\mathrm{C}(\mathrm{x}, 0)=\mathrm{C}_{0}(\mathrm{x})$ - initial conditions, concentration of feeding solution $[7,11,12]$. 
Consider the boundary conditions and their physical interpretation. Target component completely passes the boundary:

$$
C(0, t)=0,
$$

Target component does not cross the boundary:

$$
\frac{\partial C(0, t)}{\partial x}=0
$$

The flow of the target component through the membrane surface is proportional to the concentration on it:

$$
-D \frac{\partial C(0, t)}{\partial x}=\alpha_{0,1} \cdot C_{0,1}
$$

Applying the boundary conditions (13-15) in this way, the simulation of the phenomenon of concentration polarization (the formation of sediment on the membrane surface) is achieved, and the concentration $\mathrm{C}$ acts as a key parameter of the process [13, 14].

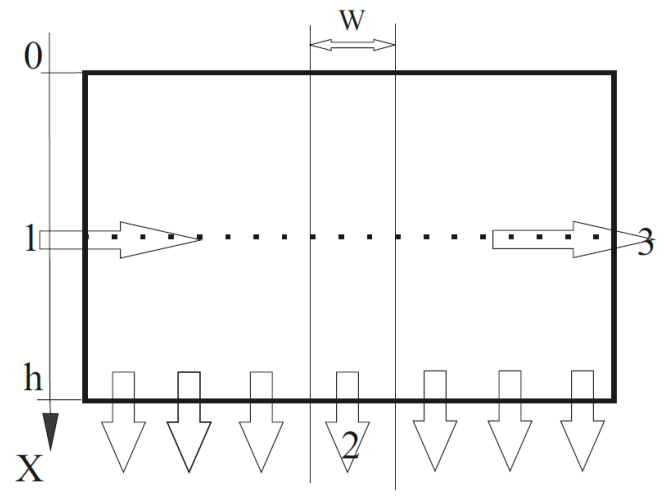

Figure 2. Scheme of the pressure channel:

1 - the flow of feeding solution; 2 - flow of permeate (filtrate); 3 - the flow of concentrate.

Consider a one-dimensional system simulating a portion of space (vertical line, with width $w \rightarrow 0$ ) of the channel of length 1 , height $\mathrm{h}$, with a membrane, whose area $\mathrm{S}$ is completely filled with a flow of solution (carrier) in which the target component is present. From the bottom there is a membrane that completely passes the carrier (solvent), but does not pass the target component (Figure 2).

For a numerical solution of equation (12), we apply the finite difference method. It is necessary to switch from continuous variables to discrete ones:

$$
\begin{gathered}
x \rightarrow x_{j}, j=0,1, \ldots, n, \\
t \rightarrow t_{i}, i=0,1, \ldots, m, \\
\Delta x=\frac{h}{n}, \\
\Delta t=\frac{t_{\max }}{m},
\end{gathered}
$$

Similarly for concentration:

$$
C(x, t) \rightarrow C\left(x_{j}, t_{i}\right)=C_{i, j},
$$


We apply marginal and initial conditions. At the initial moment of time (at $t=0)$ the concentration is equal to the concentration of feeding solution:

$$
C(x, 0)=C_{0},
$$

The key component doesn't pass through the membrane and the upper boundary of the channel:

$$
C(0, t)=C(h, t)=0,
$$

We approximate derivatives by difference schemes:

$$
\begin{gathered}
\frac{\partial C}{\partial t} \approx \frac{C_{i+1, j}-C_{i, j}}{\Delta t}, \\
D \frac{\partial^{2} C}{\partial t^{2}} \approx D \frac{C_{i, j+1}-2 C_{i, j}+C_{i, j-1}}{\Delta x^{2}} ;
\end{gathered}
$$

Consequently, by substituting expressions $(22,23)$ into equation $(11)$, we obtain a onedimensional equation in discrete variables with initial and boundary conditions:

$$
\begin{gathered}
C_{i+1, j}=C_{i, j}+D \frac{\Delta t \cdot\left(C_{i, j+1}-2 C_{i, j}+C_{i, j-1}\right)}{\Delta x^{2}}, \\
C_{0, j}=C_{0}, \\
C_{i, 0}=C_{i, n}=0,
\end{gathered}
$$

With the help of an explicit finite-difference scheme, with a given concentration value $\mathrm{C}_{\mathrm{i}, \mathrm{j}}$ at a given point, for fixed $\mathrm{i}, \mathrm{j}$ we can calculate $\mathrm{C}_{\mathrm{i}+1, \mathrm{j}}$ and thus obtain the distribution of concentrations along the entire height of the pressure channel, and its change over time.

The resulting design scheme is stable, provided that the condition of the Courant (3) [3, $5]$ is fulfilled. Based on this ratio, to ensure the stability and convergence of the circuit, the step in the direction of the axis $\mathrm{X}$ will be $0.05 \mathrm{~mm}$, respectively, the time increment is $0.01 \mathrm{~s}$ [6].

Using the calculation procedure described above (16-27), the concentration distributions by the height of the channel obtained for different values of time t (Figure 3 ). The following initial data was accepted:

1. The height of the channel $\mathrm{h}=1 \mathrm{~mm}$;

2. The area of the membrane surface $S=1 \mathrm{~m}^{2}$;

3. Convective transfer rate (velocity) $\mathrm{V}=$ const $=0.28 \mathrm{~m} / \mathrm{s}$;

4. Diffusion coefficient $\mathrm{D}=$ const $=0.05 \mathrm{~m}^{2} / \mathrm{s}$;

5. Initial concentration (feeding solution) $\mathrm{C}_{0}=2 \mathrm{~g} / \mathrm{l}$.

Estimated parameters:

1. Step by spatial coordinate $\Delta \mathrm{t}=0.05 \mathrm{~mm}$;

2. Time increment $\Delta \mathrm{t}=0.01 \mathrm{~s}$.

On Figure 3 shows how the concentration distribution by the height of channel changes over time. At the initial time (Figure 3), the target component is evenly distributed in a solution that fills the channel space (ideal mixing), but for a fairly short period of time $\Delta \mathrm{t}=512 \mathrm{sec}$, the concentration of the target component gradually increases in the region near the surface of the membrane. Distribution of concentration by the height of the channel takes an exponential character (the curve "1").

This is due to the fact that the particles of the target component are delayed on the surface of the membrane, due to which there is an increase in concentration in the membrane layer of the channel. With certain critical values of concentration, the gel sediment may be formed [12, 13, 15]. For values of dimensionless heights of the channel in the range from 0.7 to 0.9 , the concentration values coincide within $10 \%$ with the results 
presented in the works of other authors $[3,5,15]$. However, in the interval from 0.9 to 1 , the estimated concentrations are much higher than expected [5], due to assumptions and limitations of a one-dimensional model.

This is explained by the fact that in real systems, the convective flow, having a turbulent flow pattern, makes the distribution of concentrations more uniform, diverting the mass flow of the target component into the channel volume from the membrane surface. As a result, the effect of concentration polarization decreases and the sediment is formed less intensively. Consequently, the concentration at the surface of the membrane will grow at a lower intensity than the proposed model shows.

In the presented work, the convective transfer rate is assumed to be unchanged, both modulo and direction, which prevents the effects mentioned above from being taken into account. Therefore, in further work, to study the phenomena of concentration polarization, it is planned to construct a 2-dimension model based on this algorithm, taking into account the velocity distributions and effects associated with turbulence of the flow.

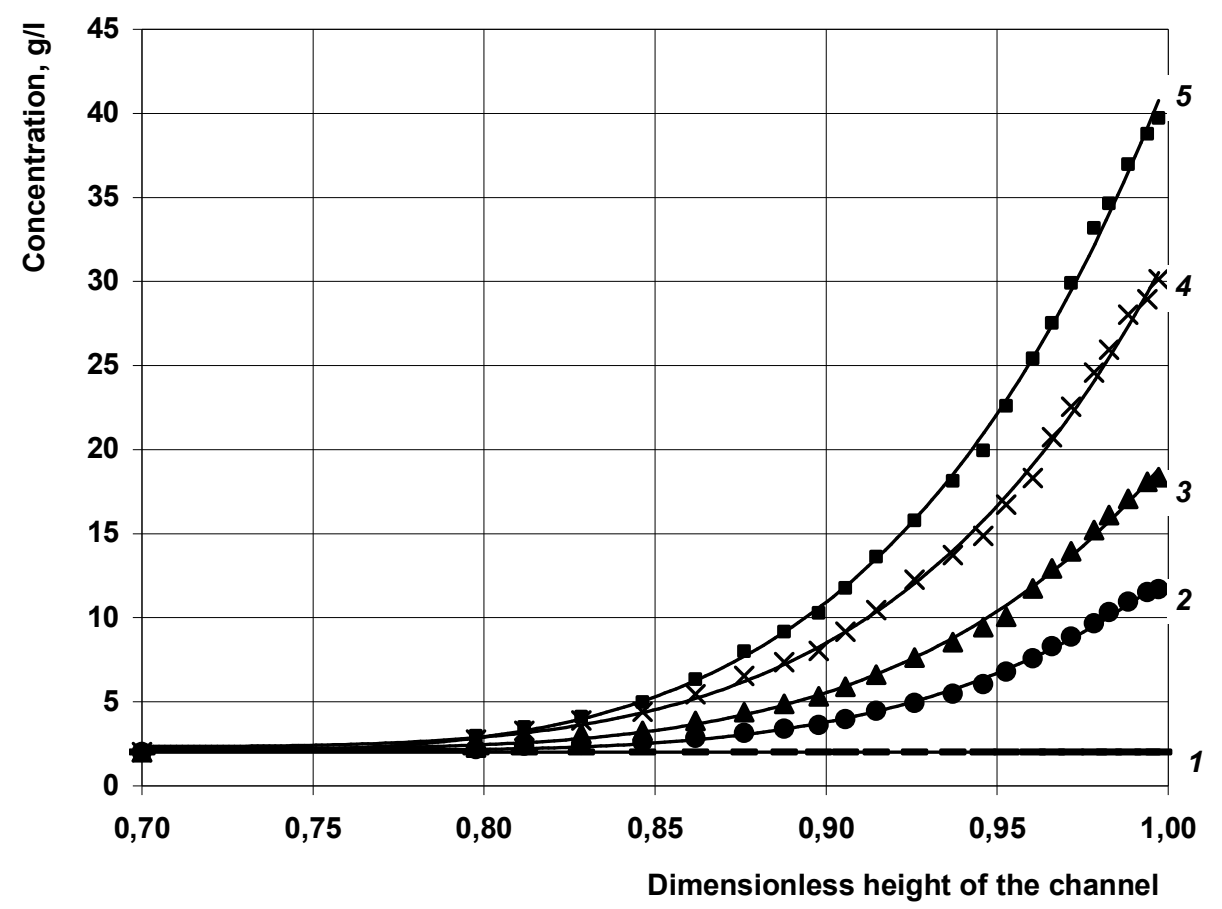

Figure 3. Distribution of concentrations by the height of the channel:

1 - 0 sec, 2 - 32 sec, 3 - 384 sec, 4 - 448 sec, 5 - 512 sec.

\section{Conclusions}

1. The distributions of concentrations of the target component by height of the membrane channel were obtained and analyzed. The character of the curves coincides with the physical representations of the process, with the exception of the region located near the surface of the membrane. This is explained by the fact that in real systems, the convective flow, having a turbulent flow pattern, makes the distribution of concentrations more uniform, diverting the mass flows of the target component into the 
volume of the channel from membrane surface. As a result, the effect of concentration polarization decreases and the sediment is formed less intensively.

2. The one-dimensional model presented does not allow for the effects associated with turbulence, but can be applied to a dead-end separation scheme.

3. The scientific novelty consists in determination of the kinetic changes in the level of concentration polarization in the membrane channels of barometric apparatus in the separation of food industry liquids.

\section{References}

1. Davydova E.B., Il'in M.I., Tarasov A.V. (2013), Simulation of the unsteady-state filtration of suspensions in a dead-end channel, Theoretical Foundations of Chemical Engineering, 47, p. 295.

2. Morao A., Nunes J.C., Sousa F., Amorim M.T.P., Escobar I.C., Queiroz J.A. (2009), Development of a model for membrane filtration of long and flexible macromolecules: application to predict dextran and linear DNA rejections in ultrafiltration, Journal of Membrane Science, 336, pp. 61-70.

3. Kumar V. (2000), Computer simulation of membrane processes: ultrafiltration and dialysis units, Computer and Chemical Engineering, 23, pp. 1713-1724.

4. Foulkes W. M. C., Mitas L., Needs R. J., Rajagopal G. (2001), Quantum Monte Carlo simulations of solids, Reviews of Modern Physics, 73, p. 33.

5. Natwar S.R., Kundariya N., Sadistap S., Narain A. (2013), Mathematical modeling and simulation of concentration polarization layer in reverse osmosis process, Engineering and System, DOI:10.1109/SCES.2013.6547547.

6. Courant R., Friedrichs K.O., Lewy H. (1928), Uber Differenzengleichungen die mathematischen Physik, Mathematsche Annalen, 100, pp. 32-74.

7. Deinega A., Valuev I. (2011), Long-time behavior of PML absorbing boundaries for layered periodic structures, Computer Physics Communications, 182, pp. 149-153.

8. Poliakov Y.S. (2014), Pore Constriction in Ultrafiltration: A Discrete Multilayer Deposition Model with Steric Exclusion of Solutes at the Pore Inlet, Theoretical Foundations of Chemical Engineering, 48(4), pp. 382-396.

9. Kim S., Hoek M.V. (2005), Modeling concentration polarization in reverse osmosis processes, Desalination, 186, pp. 111-128.

10. Ahmad A.L., Chong M.F., Bhatia S. (2005), Mathematical Modeling and simulation of the multiple solutes system for nanofiltration process, Journal of Membrane Science, 253, p. 103.

11. Lapisova K., Vlcek R., Klozova J., Rychtera M., Melzoch K. (2006), Separation techniques for distillery stillage treatment, Chez Journal of Food Science, 24, pp. 261-267.

12. Jayanti V.K., Rai P., Dasgupta S., De S. (2010), Quantification of flux decline and design of ultrafiltration system for clarification of tender coconutwater, Journal of Food Process Engineering, 33, pp. 128-143.

13. Kanani, D.M., Fissell, W.H., Roy S., Dubnisheva A., Fleischman A., Zydney A.L. (2010), Permeability-Selectivity Analysis for Ultrafiltration: Effect of Pore Geometry, Journal of Membrane Science, 349, p. 405

14. Bacchin P., Bessiere Y., Jefferson B. (2005), Dead-end filtration of natural organic matter: experimental evidence of critical conditions, Desalination, 175(1), pp. 29-36.

15. Bessiere Y., Abidine N., Bacchin P. (2005), Low fouling conditions in dead-end filtration: evidence for a critical filtered volume and interpretation using critical osmotic pressure, Journal of Membrane Science, 264, pp. 37-47. 